\title{
Secure Key-Updating for Lazy Revocation
}

\author{
Michael Backes ${ }^{1}$, Christian Cachin ${ }^{2}$, and Alina Oprea ${ }^{3}$ \\ ${ }^{1}$ Computer Science Department, Saarland University, Saarbruecken, Germany \\ backes@cs.uni-sb.de \\ ${ }^{2}$ IBM Research, Zurich Research Laboratory, Rüschlikon, Switzerland \\ cca@zurich.ibm.com \\ ${ }^{3}$ Dept. of Computer Science, Carnegie Mellon University, USA \\ alina@cs. cmu.edu
}

\begin{abstract}
We consider the problem of efficient key management and user revocation in cryptographic file systems that allow shared access to files. A performance-efficient solution to user revocation in such systems is lazy revocation, a method that delays the re-encryption of a file until the next write to that file. We formalize the notion of key-updating schemes for lazy revocation, an abstraction to manage cryptographic keys in file systems with lazy revocation, and give a security definition for such schemes. We give two composition methods that combine two secure key-updating schemes into a new secure scheme that permits a larger number of user revocations. We prove the security of two slightly modified existing constructions and propose a novel binary tree construction that is also provably secure in our model. Finally, we give a systematic analysis of the computational and communication complexity of the three constructions and show that the novel construction improves the previously known constructions.
\end{abstract}

\section{Introduction}

The recent trend of storing large amounts of data on high-speed, dedicated storage-area networks (SANs) stimulates flexible methods for information sharing, but also raises new security concerns. As the networked storage devices are subject to attacks, protecting the confidentiality of stored data is highly desirable in such an environment. Several cryptographic file systems have been designed for this purpose [15], [28], [23], [17], but practical solutions for efficient key management and user revocation still need to be developed further.

We consider cryptographic file systems that allow shared access to stored information and that use untrusted storage devices. In such systems, we can aggregate files into sets such that access permissions and ownership are managed at the level of these sets. The users who have access to the files in a set form a group, managed by the owner of the files, or the group owner. Initially, the same cryptographic key can be used to encrypt all files in a set, but upon revocation of a user from the group, the key needs to be changed to prevent access of revoked users to the files. The group owner generates and distributes this new key to the users in the group. There are two options for handling user revocation, active and lazy revocation, which differ in the way that users are revoked from a group. With active revocation, all files in a set are immediately re-encrypted with the new encryption key. The amount of work caused by a single revocation with 
this method might, however, be prohibitive for large sets of files. With the alternative method of lazy revocation, re-encryption of a file is delayed until the next write to that file and, thus, users do not experience disruptions in the operation of the file system caused by the immediate re-encryption of all files protected by the same revoked key. In systems adopting lazy revocation, the files in a set might be encrypted with different keys. Storing and distributing these keys becomes more difficult than in systems using active revocation.

In this paper, we address the problem of efficient key management in cryptographic file systems with lazy revocation. An immediate solution to this problem, adopted by the first cryptographic file systems using delayed re-encryption [15], is to store all keys for the files in a set at the group owner. However, we are interested in more efficient methods, in which the number of stored keys is not proportional to the number of revocations. We formalize the notion of key-updating schemes for lazy revocation and give a rigorous security definition. In our model, a center (e.g., the group owner) initially generates some state information, which takes the role of the master secret key. The center state is updated at every revocation. We call the period of time between two revocations a time interval. Upon a user request, the center uses its current local state to derive a user key and gives that to the user. From the user key of some time interval, a user must be able to extract the key for any previous time interval efficiently. Security for key-updating schemes requires that any polynomial-time adversary with access to the user key for a particular time interval does not obtain any information about the keys for future time intervals. The keys generated by our key-updating schemes can be used with a symmetric encryption algorithm to encrypt files for confidentiality or with a message-authentication code to authenticate files for integrity protection. Independently and concurrently to our work 1$] \mathrm{Fu}$, Kamara, and Kohno [16] have also formalized keyupdating schemes.

We describe two generic composition methods that combine two secure key updating schemes into a new scheme in which the number of time intervals is either the sum or the product of the number of time intervals of the initial schemes. Additionally, we investigate three constructions of key-updating schemes. The first scheme uses a chain of pseudorandom generator applications and is related to existing methods using one-way hash chains. It has constant update cost for the center, but the complexity of the userkey derivation is linear in the total number of time intervals. The second scheme can be based on arbitrary trapdoor permutations and generalizes the key rotation construction of the Plutus file system [23]. It has constant update and user-key derivation times, but the update algorithm uses a relatively expensive public-key operation. These two constructions require that the total number $T$ of time intervals is polynomial in the security parameter. Our third scheme uses a novel construction. It relies on a tree to derive the keys at the leaves from the master key at the root. The tree can be seen as resulting from the iterative application of the additive composition method and supports a practically unbounded number of time intervals. The binary-tree construction balances the tradeoff between the center-state update and user-key derivation algorithms (both of them have logarithmic complexity in $T$ ), at the expense of increasing the sizes of the user key and center state by a logarithmic factor in $T$.

\footnotetext{
${ }^{1}$ A preliminary version of this paper appears as [6].
} 
The rest of the paper is organized as follows. In Section 2 we give the definition of security for key-updating schemes. In Section 3 , we introduce the additive and multiplicative composition methods for secure key-updating schemes. The three constructions and proofs for their security are presented in Section 4 . A systematic analysis of the computational and communication complexities of the three constructions is given in Section 5, and an experimental evaluation is presented in Section 6. We compare our scheme to related work in Section 7

\section{Formalizing Key-Updating Schemes}

\subsection{Definition of Key-Updating Schemes}

In our model, we divide time into intervals, not necessarily of fixed length, and each time interval is associated with a new key that can be used in a symmetric-key cryptographic algorithm. In a key-updating scheme, the center generates initial state information that is updated at each time interval, and from which the center can derive a user key. The user key for interval $t$ permits a user to derive the keys of previous time intervals ( $k_{i}$ for $i \leq t$ ), but it should not give any information about keys of future time intervals $\left(k_{i}\right.$ for $\left.i>t\right)$.

We formalize key-updating schemes using the approach of modern cryptography and denote the security parameter by $\kappa$. For simplicity, we assume that all the keys are bit strings of length $\kappa$. The number of time intervals and the security parameter are given as input to the initialization algorithm.

Definition 1 (Key-Updating Schemes). A key-updating scheme consists of four deterministic polynomial time algorithms $\mathrm{KU}=$ (Init, Update, Derive, Extract) with the following properties:

- The initialization algorithm, Init, takes as input the security parameter $1^{\kappa}$, the number of time intervals $T$ and $a$ random seed $s \in\{0,1\}^{l(\kappa)}$ for a polynomial $l(\kappa)$, and outputs a bit string $S_{0}$, called the initial center state.

- The key update algorithm, Update, takes as input the current time interval $0 \leq t \leq$ $T-1$, the current center state $S_{t}$, and outputs the center state $S_{t+1}$ for the next time interval.

- The user key derivation algorithm, Derive, is given as input a time interval $1 \leq t \leq$ $T$ and the center state $S_{t}$, and outputs the user key $M_{t}$. The user key can be used to derive all keys $k_{i}$ for $1 \leq i \leq t$.

- The key extraction algorithm, Extract, is executed by the user and takes as input a time interval $1 \leq t \leq T$, the user key $M_{t}$ for interval $t$ as received from the center, and $a$ target time interval $i$ with $1 \leq i \leq t$. The algorithm outputs the key $k_{i}$ for interval $i$.

W.l.o.g., we assume that the Update algorithm is run at least once after the Init algorithm, before any user keys can be derived. The first time the Update algorithm is run, it is given as input time interval $t=0$. User keys and keys are associated with the time intervals between 1 and $T$. 


\subsection{Applications to Cryptographic File Systems}

In a cryptographic file system adopting lazy revocation, the re-encryption of a file after a revocation is delayed until the next write to that file. Similarly to the Plutus file system, files can be divided into sets based on their access permissions, such that all files in a set have the same permissions. Initially, all files in a set can be encrypted with the same key. We assume that file owners are responsible for the generation and distribution of keys to the authorized users, so file owners take the role of the center in our model of key-updating schemes.

When a user is revoked from the group of users having access to the set of files, the file owner runs the Update algorithm generating a new state and advancing the time interval. The file owner then runs Derive and the new user key is distributed to all the users that have now access permissions to the files. A user writing a file uses the encryption key for the latest time interval, which can be efficiently extracted from the latest user key. To decrypt a file, a user needs to know the version of the key that was used to encrypt it, and extract the appropriate encryption key from the user key. The key version with which each file is encrypted might, for example, be stored in the file i-node on the file server.

Assuming that the integrity of files is protected with a message-authentication code (MAC), key-updating schemes can also be used to manage symmetric keys for authentication. To guarantee independence of the keys used for confidentiality and integrity, different instances of key-updating schemes have to be used for encryption and authentication.

\subsection{Security of Key-Updating Schemes}

The definition of security for key-updating schemes requires that a polynomial-time adversary with access to the user key for a time interval $t$ is not able to derive any information about the keys for the next time interval. The definition we give here is related to the definition of forward-secure pseudorandom generators given by Bellare and Yee [8]. Formally, consider a probabilistic polynomial-time adversary $\mathcal{A}=\left(\mathcal{A}_{\mathcal{U}}, \mathcal{A}_{\mathcal{G}}\right)$ that participates in the following experiment:

Initialization: The initial center state is generated with the Init algorithm.

Key updating: The adversary adaptively picks a time interval $t$ such that $0 \leq t \leq T-1$ as follows. Starting with $t=0,1, \ldots$, algorithm $\mathcal{A}_{\mathcal{U}}$ is given the user keys $M_{t}$ for all consecutive time intervals until $\mathcal{A}_{\mathcal{U}}$ decides to output stop or $t$ becomes equal to $T-1$. We require that $\mathcal{A}_{\mathcal{U}}$, a probabilistic polynomial-time algorithm, outputs stop at least once before halting. $\mathcal{A}_{\mathcal{U}}$ also outputs some additional information $z \in$ $\{0,1\}^{*}$ that is given as input to algorithm $\mathcal{A}_{\mathcal{G}}$.

Challenge: A challenge for the adversary is generated, which is either the key for time interval $t+1$ generated with the Update, Derive and Extract algorithms, or a random bit string of length $\kappa$.

Guess: $\mathcal{A}_{\mathcal{G}}$ takes the challenge and $z$ as inputs and outputs a bit $b$.

The key-updating scheme is secure if the advantage of the adversary of distinguishing between the properly generated key for time interval $t+1$ and the random key is only negligibly larger than $\frac{1}{2}$. More formally, the definition of a secure key-updating scheme is the following: 
Definition 2 (Security of Key-Updating Schemes). Let KU = (Init, Update, Derive, Extract) be a key-updating scheme and $\mathcal{A}$ a polynomial-time adversary algorithm that participates in one of the two experiments defined in Figure 1$]$

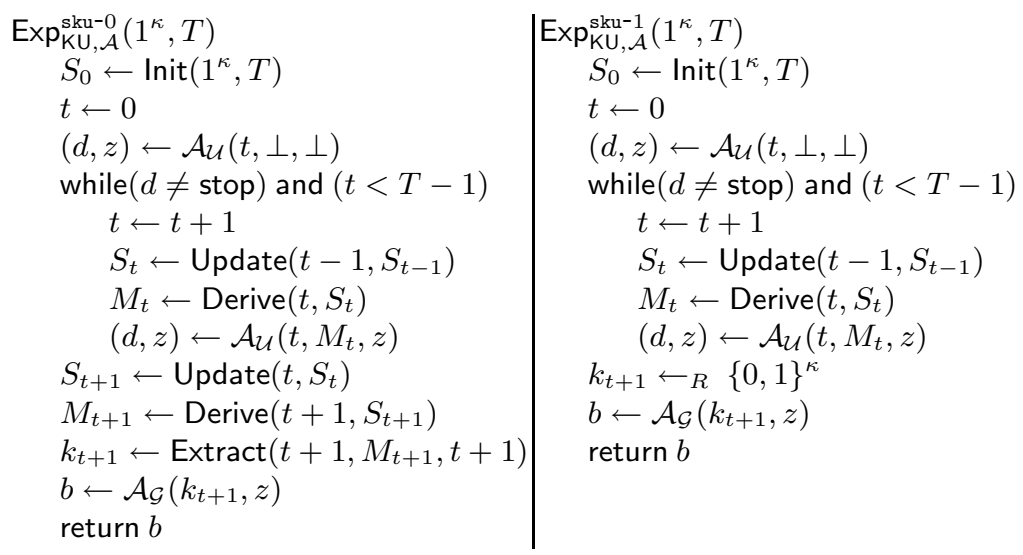

Fig. 1. Experiments defining the security of key-updating schemes

The advantage of the adversary $\mathcal{A}=\left(\mathcal{A}_{\mathcal{U}}, \mathcal{A}_{\mathcal{G}}\right)$ for $\mathrm{KU}$ is defined as

$$
\operatorname{Adv}_{\mathrm{KU}, \mathcal{A}}^{\mathrm{sku}}(\kappa, T)=\left|\operatorname{Pr}\left[\operatorname{Exp}_{\mathrm{KU}, \mathcal{A}}^{\mathrm{sku}-1}\left(1^{\kappa}, T\right)=1\right]-\operatorname{Pr}\left[\operatorname{Exp}_{\mathrm{KU}, \mathcal{A}}^{\mathrm{sku}-0}\left(1^{\kappa}, T\right)=1\right]\right| .
$$

Without loss of generality, we can relate the success probability of adversary $\mathcal{A}$ of distinguishing between the two experiments and its advantage as

$$
\operatorname{Pr}[\mathcal{A} \text { succeeds }]=\frac{1}{2}\left[1+\operatorname{Adv}_{\mathrm{KU}, \mathcal{A}}^{\mathrm{sku}}(\kappa, T)\right] .
$$

The key-updating scheme $\mathrm{KU}$ is secure if for all polynomial-time adversaries $\mathcal{A}$ and all $T$, there exists a negligible function $\epsilon$ such that $\operatorname{Adv}_{\mathrm{KU}, \mathcal{A}}^{\mathrm{sku}}(\kappa, T)=\epsilon(\kappa)$.

Remark. The security notion we have defined is equivalent to a seemingly stronger security definition, in which the adversary can choose the challenge time interval $t^{*}$ with the restriction that $t^{*}$ is greater than the time interval at which the adversary outputs stop and that $t^{*}$ is polynomial in the security parameter. This second security definition guarantees, intuitively, that the adversary is not gaining any information about the keys of any future time intervals after it outputs stop.

\section{Composition of Key-Updating Schemes}

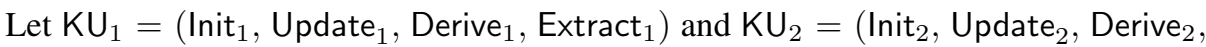
Extract $_{2}$ ) be two secure key-updating schemes using the same security parameter $\kappa$ with $T_{1}$ and $T_{2}$ time intervals, respectively. In this section, we show how to combine the two schemes into a secure key-updating scheme $K U=$ (Init, Update, Derive, Extract), 
which is either the additive or multiplicative composition of the two schemes with $T=$ $T_{1}+T_{2}$ and $T=T_{1} \cdot T_{2}$ time intervals, respectively. Similar generic composition methods have been given previously for forward-secure signature schemes [26].

For simplicity, we assume the length of the random seed in the Init algorithm of the scheme KU to be $\kappa$ for both composition methods. Let $G:\{0,1\}^{\kappa} \rightarrow\{0,1\}^{l_{1}(\kappa)+l_{2}(\kappa)}$ be a pseudorandom generator; it can be used to expand a random seed of length $\kappa$ into two random bit strings of length $l_{1}(\kappa)$ and $l_{2}(\kappa)$, respectively, as needed for Init I $_{1}$ and Init 2 . We write $G(s)=G_{1}(s) \| G_{2}(s)$ with $\left|G_{1}(s)\right|=l_{1}(\kappa)$ and $\left|G_{2}(s)\right|=l_{2}(\kappa)$ for $s \in\{0,1\}^{\kappa}$.

\subsection{Additive Composition}

The additive composition of two key-updating schemes uses the keys generated by the first scheme for the first $T_{1}$ time intervals and the keys generated by the second scheme for the subsequent $T_{2}$ time intervals. The user key for the first $T_{1}$ intervals in $\mathrm{KU}$ is the same as that of scheme $\mathrm{KU}_{1}$ for the same interval. For an interval $t$ greater than $T_{1}$, the user key includes both the user key for interval $t-T_{1}$ of scheme $\mathrm{KU}_{2}$, and the user key for interval $T_{1}$ of scheme $\mathrm{KU}_{1}$. The details of the additive composition method are described in Figure 2. The security of the composition operation is analyzed in the following theorem, whose proof is given in the full version of this paper [6].

Theorem 1. Suppose that $\mathrm{KU}_{1}=\left(\right.$ Init $_{1}$, Update ${ }_{1}$, Derive ${ }_{1}$, Extract $\left.{ }_{1}\right)$ and $\mathrm{KU}_{2}=$ (Init ${ }_{2}$, Update $_{2}$, Derive 2 , Extract ${ }_{2}$ ) are two secure key-updating schemes with $T_{1}$ and $\mathrm{T}_{2}$ time intervals, respectively, and that $G$ is a pseudorandom generator as above. Then $\mathrm{KU}=$ (Init, Update, Derive, Extract) described in Figure 2 denoted as $\mathrm{KU}_{1} \oplus \mathrm{KU}_{2}$ is a secure key-updating scheme with $T_{1}+T_{2}$ time intervals.

\begin{tabular}{|c|c|}
\hline Init $\left(1^{\kappa}, T, s\right)$ & Derive $\left(t,\left(S_{t}^{1}, S_{t}^{2}\right)\right)$ \\
$S_{0}^{1} \leftarrow \operatorname{Init}_{1}\left(1^{\kappa}, T_{1}, G_{1}(s)\right)$ & if $t<T_{1}$ \\
$S_{0}^{2} \leftarrow \operatorname{Init}_{2}\left(1^{\kappa}, T_{2}, G_{2}(s)\right)$ & $M_{t}^{1} \leftarrow \operatorname{Derive}_{1}\left(t, S_{t}^{1}\right)$ \\
return $\left(S_{0}^{1}, S_{0}^{2}\right)$ & $M_{t}^{2} \leftarrow \perp$ \\
& else $M_{t}^{1} \leftarrow \operatorname{Derive}_{1}\left(T_{1}, S_{t}^{1}\right)$ \\
& $M_{t}^{2} \leftarrow \operatorname{Derive}_{2}\left(t-T_{1}, S_{t}^{2}\right)$ \\
& return $\left(M_{t}^{1}, M_{t}^{2}\right)$ \\
\hline $\begin{array}{c}\text { Update }\left(t,\left(S_{t}^{1}, S_{t}^{2}\right)\right) \\
\text { if } t<T_{1}\end{array}$ & Extract $\left(t,\left(M_{t}^{1}, M_{t}^{2}\right), i\right)$ \\
$S_{t+1}^{1} \leftarrow \operatorname{Update}_{1}\left(t, S_{t}^{1}\right)$ & if $i>T_{1}$ \\
$S_{t+1}^{2} \leftarrow S_{t}^{2}$ & $k_{i} \leftarrow \operatorname{Extract}_{2}\left(t-T_{1}, M_{t}^{2}, i-T_{1}\right)$ \\
else & else $t<T_{1}$ \\
$S_{t+1}^{1} \leftarrow S_{t}^{1}$ & if $t \leftarrow \operatorname{Extract}_{1}\left(t, M_{t}^{1}, i\right)$ \\
$S_{t+1}^{2} \leftarrow \mathrm{Update}_{2}\left(t-T_{1}, S_{t}^{2}\right)$ & else \\
return $\left(S_{t+1}^{1}, S_{t+1}^{2}\right)$ & $k_{i} \leftarrow \operatorname{Extract}_{1}\left(T_{1}, M_{t}^{1}, i\right)$ \\
& return $k_{i}$ \\
\hline
\end{tabular}

Fig. 2. The additive composition of $\mathrm{KU}_{1}$ and $\mathrm{KU}_{2}$ 
Extended Additive Composition. It is immediate to extend the additive composition to construct a new scheme with $T_{1}+T_{2}+1$ time intervals. The idea is to use the first scheme for the keys of the first $T_{1}$ intervals, the second scheme for the keys of the next $T_{2}$ intervals, and the seed $s$ as the key for the $\left(T_{1}+T_{2}+1\right)$-th interval. By revealing the seed $s$ as the user key at interval $T_{1}+T_{2}+1$, all previous keys of $\mathrm{KU}_{1}$ and $\mathrm{KU}_{2}$ can be derived. This idea will be useful in our later construction of a binary tree key-updating scheme. We call this composition method extended additive composition.

\subsection{Multiplicative Composition}

The idea behind the multiplicative composition operation is to use every key of the first scheme to seed an instance of the second scheme. Thus, for each one of the $T_{1}$ time intervals of the first scheme, we generate an instance of the second scheme with $T_{2}$ time intervals.

We denote a time interval $t$ for $1 \leq t \leq T_{1} \cdot T_{2}$ of scheme $\mathrm{KU}$ as a pair $t=\langle i, j\rangle$, where $i$ and $j$ are such that $t=(i-1) T_{2}+j$ for $1 \leq i \leq T_{1}$ and $1 \leq j \leq T_{2}$. The Update algorithm is run initially for time interval $t=0$, which will be expressed as $<0,0>$. The user key for a time interval $t=\langle i, j\rangle$ includes both the user key for time interval $i-1$ of scheme $\mathrm{KU}_{1}$ and the user key for time interval $j$ of scheme $\mathrm{KU}_{2}$. A user receiving $M_{<i, j>}$ can extract the key for any time interval $\left.\langle m, n\rangle \leq<i, j\right\rangle$ by first extracting the key $K$ for time interval $m$ of $\mathrm{KU}_{1}$ (this step needs to be performed only if $m<i$ ), then using $K$ to derive the initial state of the $m$-th instance of the scheme $\mathrm{KU}_{2}$, and finally, deriving the key $k_{<m, n>}$. The details of the multiplicative composition method are shown in Figure 3.

\begin{tabular}{|c|c|}
\hline $\begin{array}{l}\operatorname{Init}\left(1^{\kappa}, T, s\right) \\
\quad S_{0}^{1} \leftarrow \operatorname{Init}_{1}\left(1^{\kappa}, T_{1}, G_{1}(s)\right) \\
S_{1}^{1} \leftarrow \operatorname{Update}_{1}\left(0, S_{0}^{1}\right) \\
\quad k_{1}^{1} \leftarrow \operatorname{Extract}_{1}\left(1, \operatorname{Derive}_{1}\left(1, S_{1}^{1}\right), 1\right) \\
\quad S_{0}^{2} \leftarrow \operatorname{Init}_{2}\left(1^{\kappa}, T_{2}, G_{2}\left(k_{1}^{1}\right)\right) \\
\quad \text { return }\left(\perp, S_{0}^{1}, S_{0}^{2}\right)\end{array}$ & $\begin{array}{l}\text { Derive }\left(<i, j>,\left(S_{i-1}^{1}, S_{i}^{1}, S_{j}^{2}\right)\right) \\
\quad \text { if } i>1 \\
\quad M_{i-1}^{1} \leftarrow \operatorname{Derive}_{1}\left(i-1, S_{i-1}^{1}\right) \\
\text { else } \\
\quad M_{i-1}^{1} \leftarrow \perp \\
\quad M_{j}^{2} \leftarrow \operatorname{Derive}_{2}\left(j, S_{j}^{2}\right) \\
\quad \text { return }\left(M_{i-1}^{1}, M_{j}^{2}\right)\end{array}$ \\
\hline $\begin{array}{l}\text { Update }\left(<i, j>,\left(S_{i-1}^{1}, S_{i}^{1}, S_{j}^{2}\right)\right) \\
\text { if } j=T_{2} \\
S_{i+1}^{1} \leftarrow \operatorname{Update}_{1}\left(i, S_{i}^{1}\right) \\
k_{i+1}^{1} \leftarrow \operatorname{Extract}_{1}(i+1, \\
\left.\quad \operatorname{Derive}_{1}\left(i+1, S_{i+1}^{1}\right), i+1\right) \\
S_{0}^{2} \leftarrow \operatorname{Init}_{2}\left(1^{\kappa}, T_{2}, G_{2}\left(k_{i+1}^{1}\right)\right) \\
S_{1}^{2} \leftarrow \operatorname{Update}_{2}\left(0, S_{0}^{2}\right) \\
\quad \text { return }\left(S_{i}^{1}, S_{i+1}^{1}, S_{1}^{2}\right) \\
\text { else } \\
\quad S_{j+1}^{2} \leftarrow \operatorname{Update}_{2}\left(j, S_{j}^{2}\right) \\
\quad \text { return }\left(S_{i-1}^{1}, S_{i}^{1}, S_{j+1}^{2}\right)\end{array}$ & $\begin{array}{l}\text { Extract }\left(<i, j>,\left(M_{i-1}^{1}, M_{j}^{2}\right),<m, n>\right) \\
\quad \text { if } i=m \\
\quad k_{<m, n>} \leftarrow \operatorname{Extract}_{2}\left(j, M_{j}^{2}, m\right) \\
\text { else } \\
\quad K \leftarrow \operatorname{Extract}_{1}\left(i-1, M_{i-1}^{1}, m\right) \\
\quad S_{0}^{2} \leftarrow \operatorname{Init}_{2}\left(1^{\kappa}, T_{2}, G_{2}(K)\right) \\
\quad k_{<m, n>} \leftarrow \operatorname{Extract}_{2}\left(T_{2}, S_{0}^{2}, n\right) \\
\text { return } k_{<m, n>}\end{array}$ \\
\hline
\end{tabular}

Fig. 3. The multiplicative composition of $\mathrm{KU}_{1}$ and $\mathrm{KU}_{2}$ 
The security of the multiplicative composition method is analyzed in the following theorem, whose proof is given in the full version of this paper [6].

Theorem 2. Suppose that $\mathrm{KU}_{1}=\left(\right.$ Init $_{1}$, Update ${ }_{1}$, Derive ${ }_{1}$, Extract $\left.{ }_{1}\right)$ and $\mathrm{KU}_{2}=$ (Init ${ }_{2}$, Update $_{2}$, Derive 2 , Extract ${ }_{2}$ ) are two secure key-updating schemes with $T_{1}$ and $T_{2}$ time intervals, respectively, and that $G$ is a pseudorandom generator as above. Then $\mathrm{KU}=$ (Init, Update, Derive, Extract) described in Figure 3 denoted as $\mathrm{KU}_{1} \otimes \mathrm{KU}_{2}$ is a secure key-updating scheme with $T_{1} \cdot T_{2}$ time intervals.

\section{Constructions}

In this section, we describe three constructions of key-updating schemes with different complexity and communication tradeoffs. The first two constructions are based on previously proposed methods [23 16]. We give cryptographic proofs that demonstrate the security of the existing constructions after some subtle modifications. Additionally, we propose a third construction that is more efficient than the known schemes. It uses a binary tree to derive the user keys and is also provably secure in our model.

\subsection{Chaining Construction (CKU)}

In this construction, the center generates an initial random seed of length $\kappa$ and applies a pseudorandom generator iteratively $i$ times to obtain the key for time interval $T-i$, for $1 \leq i \leq T-1$. This construction is inspired by a folklore method using a hash chain for deriving the keys. A construction based on a hash chain can be proven secure if the hash function $h$ is modeled as a random oracle. To obtain a provably secure scheme in the standard model, we replace the hash function with a pseudorandom generator.

Let $G:\{0,1\}^{\kappa} \rightarrow\{0,1\}^{2 \kappa}$ be a pseudorandom generator. We write $G(s)=$ $G_{1}(s) \| G_{2}(s)$ with $\left|G_{1}(s)\right|=\left|G_{2}(s)\right|=\kappa$ for $s \in\{0,1\}^{\kappa}$. The algorithms of the chaining construction, called CKU, are:

- Init $\left(1^{\kappa}, T, s\right)$ generates a random seed $s_{0}$ of length $\kappa$ from $s$ and outputs $S_{0}=s_{0}$.

- Update $\left(t, S_{t}\right)$ copies the state $S_{t}$ into $S_{t+1}$.

- Derive $\left(t, S_{t}\right)$ and Extract $\left(t, M_{t}, i\right)$ are given in Figure 4

\begin{tabular}{|c|c|}
\hline Derive $\left(t, S_{t}\right)$ & Extract $\left(t, M_{t}, i\right)$ \\
$B_{T+1} \leftarrow S_{t}$ & $\left(B_{t}, k_{t}\right) \leftarrow M_{t}$ \\
for $i=T$ downto $t$ & for $j=t-1$ downto $i$ \\
$\quad\left(B_{i}, k_{i}\right) \leftarrow G\left(B_{i+1}\right)$ & $\left(B_{j}, k_{j}\right) \leftarrow G\left(B_{j+1}\right)$ \\
return $\left(B_{t}, k_{t}\right)$ & return $k_{i}$ \\
\hline
\end{tabular}

Fig. 4. The Derive $\left(t, S_{t}\right)$ and Extract $\left(t, M_{t}, i\right)$ algorithms of the chaining construction

This construction has constant center-state size and linear cost for the user-key derivation algorithm. An alternative construction with linear center-state size and constant user-key derivation is to precompute all the keys $k_{i}$ and user keys $M_{i}$, for $1 \leq i \leq T$ in the Init algorithm and store all of them in the initial center state $S_{0}$. The security of the chaining construction is given be the following theorem, whose proof is in the full version of this paper [6]. 
Theorem 3. Given a pseudorandom generator G, CKU is a secure key-updating scheme.

\subsection{Trapdoor Permutation Construction (TDKU)}

In this construction, the center picks an initial random state that is updated at each time interval by applying the inverse of a trapdoor permutation. The trapdoor is known only to the center, but a user, given the state at a certain moment, can apply the permutation iteratively to generate all previous states. The key for a time interval is generated by applying a hash function, modeled as a random oracle, to the current state. This idea underlies the key rotation mechanism of the Plutus file system [23], with the difference that Plutus uses the output of an RSA trapdoor permutation directly for the encryption key. We could not prove the security of this scheme in our model for key-updating schemes, even when the trapdoor permutation is not arbitrary, but instantiated with the RSA permutation.

This construction has the advantage that knowledge of the total number of time intervals is not needed in advance; on the other hand, its security can only be proved in the random oracle model. Let a family of trapdoor permutations be given such that the domain size of the permutations with security parameter $\kappa$ is $l(\kappa)$, for some polynomial $l$. Let $h:\{0,1\}^{l(\kappa)} \rightarrow\{0,1\}^{\kappa}$ be a hash function modeled as a random oracle. The detailed construction of the trapdoor permutation scheme, called TDKU, is presented below:

- Init $\left(1^{\kappa}, T, s\right)$ generates a random $s_{0} \leftarrow_{R}\{0,1\}^{l(\kappa)}$ and a trapdoor permutation $f:\{0,1\}^{l(\kappa)} \rightarrow\{0,1\}^{l(\kappa)}$ with trapdoor $\tau$ from seed $s$ using a pseudorandom generator. Then it outputs $S_{0}=\left(s_{0}, f, \tau\right)$.

- Update $\left(t, S_{t}\right)$ with $S_{t}=\left(s_{t}, f, \tau\right)$ computes $s_{t+1}=f^{-1}\left(s_{t}\right)$ and outputs $S_{t+1}=$ $\left(s_{t+1}, f, \tau\right)$.

- Derive $\left(t, S_{t}\right)$ outputs $M_{t} \leftarrow\left(s_{t}, f\right)$.

- Extract $\left(t, M_{t}, i\right)$ applies the permutation iteratively $t-i$ times to generate state $s_{i}=f^{t-i}\left(M_{t}\right)$ and then outputs $h\left(s_{i}\right)$.

The security of this construction is given be the following theorem, whose proof is in the full version of this paper [6].

Theorem 4. Given a family of trapdoor permutations and a hash function $h$, TDKU is a secure key-updating scheme in the random oracle model.

\subsection{Tree Construction (TreeKU)}

In the two schemes above, at least one of the algorithms Update, Derive and Extract has worst-case complexity linear in the total number of time intervals. We present a tree construction based on ideas of Canetti, Halevi and Katz [10] with constant complexity for the Derive algorithm and logarithmic worst-case complexity in the number of time intervals for the Update and Extract algorithms. Moreover, the amortized complexity of the Update algorithm is constant. In this construction, the user key size is increased by at most a logarithmic factor in $T$ compared to the user key size of the two constructions described above. 
Our tree-based key-updating scheme, called TreeKU, generates keys using a complete binary tree with $T$ nodes, assuming that $T=2^{d}-1$ for some $d \in \mathbb{Z}$. Each node in the tree is associated with a time interval between 1 and $T$, a unique label in $\{0,1\}^{*}$, a tree-key in $\{0,1\}^{\kappa}$ and an external key in $\{0,1\}^{\kappa}$ such that:

1. Time intervals are assigned to tree nodes using post-order tree traversal, i.e., a node corresponds to interval $i$ if it is the $i$-th node in the post-order traversal of the tree. We refer to the node associated with interval $t$ as node $t$.

2. We define a function label that maps node $t$ with $1 \leq t \leq T$ to its label in $\{0,1\}^{*}$ as follows. The root of the tree is labeled by the empty string $\varepsilon$, and the left and right children of a node with label $\ell$ are labeled by $\ell \| 0$ and by $\ell \| 1$, respectively. The parent of a node with label $\ell$ is denoted by parent $(\ell)$, thus parent $(\ell \| 0)=$ parent $(\ell \| 1)=\ell$. We denote the length of a label $\ell$ by $|\ell|$.

3. The tree-key for the root node is chosen at random. The tree-keys for the two children of an internal node in the tree are derived from the tree-key of the parent node using a pseudorandom generator $G:\{0,1\}^{\kappa} \rightarrow\{0,1\}^{2 \kappa}$. For an input $s \in\{0,1\}^{\kappa}$, we write $G(s)=G_{1}(s) \| G_{2}(s)$ with $\left|G_{1}(s)\right|=\left|G_{2}(s)\right|=\kappa$. If the tree-key for the internal node with label $\ell$ is denoted $u_{\ell}$, then the tree-keys for its left and right children are $u_{\ell \| 0}=G_{1}\left(u_{\ell}\right)$ and $u_{\ell \| 1}=G_{2}\left(u_{\ell}\right)$, respectively. This implies that once the tree-key for a node is revealed, then the tree-keys of its children can be computed, but knowing the tree-keys of both children of a node does not reveal any information about the tree-key of the node.

4. The external key of a node $t$ is the key $k_{t}$ output by the scheme to the application for interval $t$. For a node $t$ with tree-key $u_{\text {label }(t)}$, the external key $k_{t}$ is obtained by computing $F_{u_{\text {label }(t)}}(1)$, where $F_{u}(b)=F(u, b)$ and $F:\{0,1\}^{\kappa} \times\{0,1\} \rightarrow\{0,1\}^{\kappa}$ is a pseudorandom function on bits.

We describe the four algorithms of the binary tree key-updating scheme:

- Init $\left(1^{\kappa}, T, s\right)$ generates the tree-key for the root node randomly, $u_{T} \leftarrow_{R}\{0,1\}^{\kappa}$, using seed $s$, and outputs $S_{0}=\left(\left\{\left(\varepsilon, u_{T}\right)\right\}, \emptyset\right)$.

- Update $\left(t, S_{t}\right)$ updates the state $S_{t}=\left(P_{t}, L_{t}\right)$ to the next center state $S_{t+1}=$ $\left(P_{t+1}, L_{t+1}\right)$. The center state for interval $t$ consists of two sets: $P_{t}$ that contains pairs of (label, tree-key) for all nodes on the path from the root to node $t$ (including node $t$ ), and $L_{t}$ that contains label/tree-key pairs for all left siblings of the nodes in $P_{t}$ that are not in $P_{t}$.

We use several functions in the description of the Update algorithm. For a label $\ell$ and a set $A$ of label/tree-key pairs, we define a function searchkey $(\ell, A)$ that outputs a tree-key $u$ for which $(\ell, u) \in A$, if the label exists in the set, and $\perp$ otherwise. Given a label $\ell$ and a set of label/tree-key pairs $A$, function rightsib $(\ell, A)$ returns the label and the tree-key of the right sibling of the node with label $\ell$, and, similarly, function leftsib $(\ell, A)$ returns the label and the tree-key of the left sibling of the node with label $\ell$ (assuming the labels and tree-keys of the siblings are in $A$ ). The function leftkeys is given as input a label/tree-key pair of a node and returns all label/tree-key pairs of the left-most nodes in the subtree rooted at the input node, including label and tree-key of the input node. 


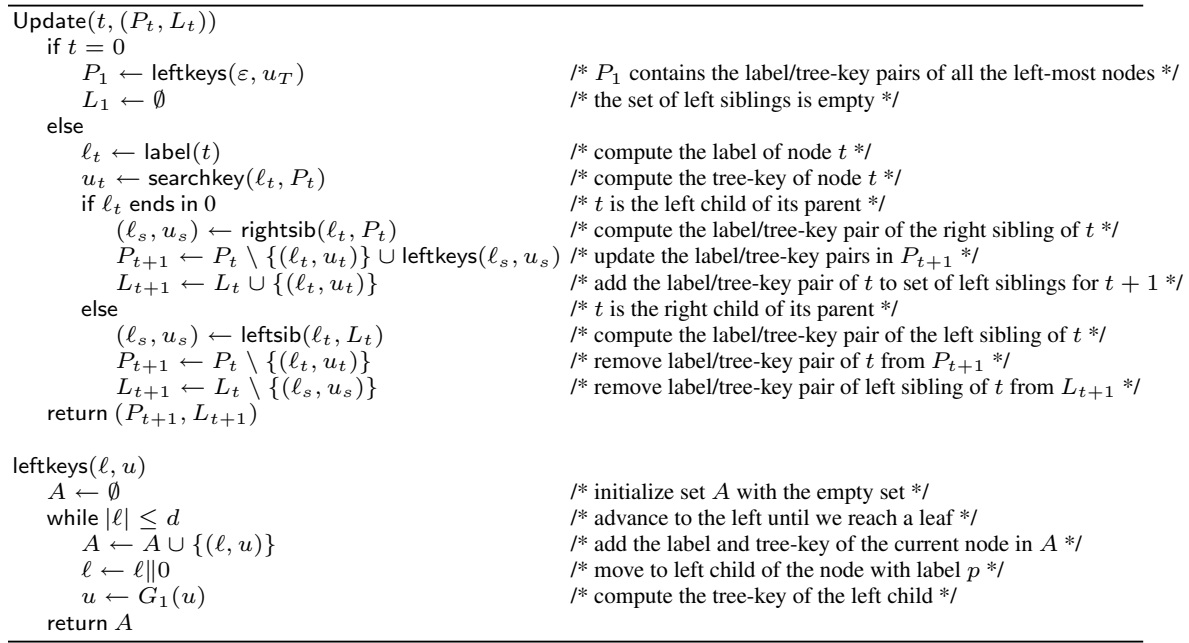

Fig. 5. The Update $\left(t,\left(P_{t}, L_{t}\right)\right)$ algorithm

The code for the Update and leftkeys algorithms is given in Figure 5. We omit the details of functions searchkey, rightsib and leftsib. The Update algorithm distinguishes three cases:

1. If $t=0$, the Update algorithm computes the label/tree-key pairs of all leftmost nodes in the complete tree using function leftkeys and stores them in $P_{1}$. The set $L_{1}$ is empty in this case, as nodes in $P_{1}$ do not have left siblings.

2. If $t$ is the left child of its parent, the successor of node $t$ in post-order traversal is the left-most node in the subtree rooted at the right sibling $t^{\prime}$ of node $t$. $P_{t+1}$ contains all label/tree-key pairs in $P_{t}$ except the tuple for node $t$, and, in addition, all label/tree-key pairs for the left-most nodes in the subtree rooted at $t^{\prime}$, which are computed by leftkeys. The set of left siblings $L_{t+1}$ contains all label/tree-key pairs from $L_{t}$ and, in addition, the label/tree-key pair for node $t$.

3. If $t$ is the right child of its parent, node $t+1$ is its parent, so $P_{t+1}$ contains all label/tree-key pairs from $P_{t}$ except the tuple for node $t$, and $L_{t+1}$ contains all the label/tree-key pairs in $L_{t}$ except the pair for the left sibling of node $t$.

- Algorithm Derive $\left(t,\left(P_{t}, L_{t}\right)\right)$ outputs the user tree-key $M_{t}$, which is the minimum information needed to generate the set of tree-keys $\left\{u_{i}: i \leq t\right\}$. Since the treekey of any node reveals the tree-keys for all nodes in the subtree rooted at that node, $M_{t}$ consists of the label/tree-key pairs for the left siblings (if any) of all nodes on the path from the root to the parent of node $t$ and the label/tree-key pair of node $t$. This information has already been pre-computed such that one can set $M_{t} \leftarrow\left\{\left(\operatorname{label}(t), u_{t}\right)\right\} \cup L_{t}$.

- Algorithm Extract $\left(t, M_{t}, i\right)$ first finds the maximum predecessor of node $i$ in postorder traversal whose label/tree-key pair is included in the user tree-key $M_{t}$. Then it computes the tree-keys for all nodes on the path from that predecessor to node $i$. The external key $k_{i}$ is derived from the tree-key $u_{i}$ as $k_{i}=F_{u_{i}}(1)$ using the pseudorandom function. The algorithm is in Figure 6 


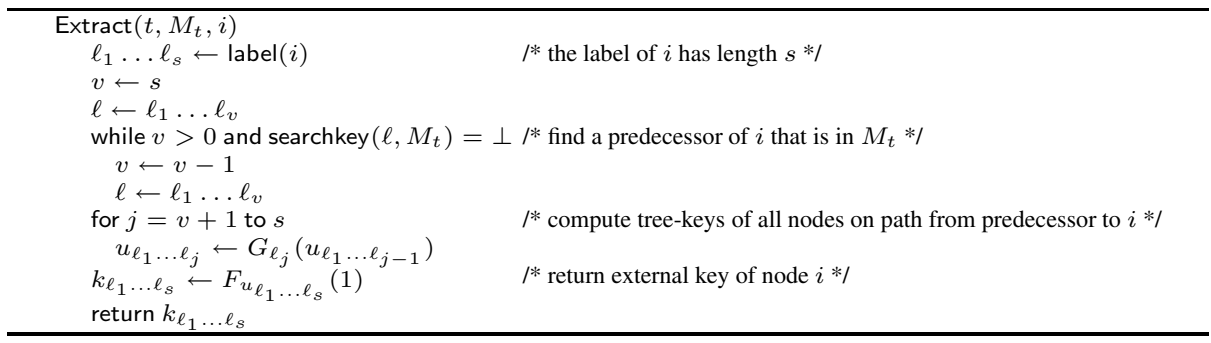

Fig. 6. The Extract $\left(t, M_{t}, i\right)$ algorithm

Analysis of Complexity. The worst-case complexity of the cryptographic operations used in the Update and Extract algorithms is logarithmic in the number of time intervals, and that of Derive is constant. However, it is easy to see that the key for each node is computed exactly once if $T$ updates are executed. This implies that the total cost of all update operations is $T$ pseudorandom-function applications, so the amortized cost per update is constant. The size of the center state and the user key is proportional to the height of the binary tree, so the worst-case space complexity is $\mathcal{O}\left(\kappa \log _{2} T\right)$ bits.

The security of the tree construction is given be the following theorem, whose proof is in the full version of this paper [6].

Theorem 5. Given a pseudorandom generator $G$ and a pseudorandom function $F$, TreeKU is a secure key-updating scheme.

An Incremental Tree Construction. We can construct an incremental tree scheme using ideas from the generic forward-secure signature scheme of Malkin, Micciancio, and Miner [26]. The incremental scheme does not require the total number of time intervals to be known in advance.

Let TreeKU $(i)$ be the binary tree construction with $2^{i}-1$ nodes. Then the incremental tree scheme is obtained by additively composing binary tree schemes with increasing number of intervals: TreeKU(1) $\oplus \operatorname{TreeKU}(2) \oplus \operatorname{TreeKU}(3) \oplus \ldots$ The keys generated by the tree scheme $\operatorname{TreeKU}(i)$ correspond to the time intervals between $2^{i}-i$ and $2^{i+1}-i-2$ in the incremental scheme. Once the intervals of the tree scheme TreeKU $(i)$ are exhausted, an instance of $\operatorname{TreeKU}(i+1)$ is generated, if needed.

In addition to allowing a practically unbounded number of time intervals, this construction has the property that the complexity of the Update, Derive and Extract algorithms and the size of the center state and user key depend on the number of past time intervals. Below we perform a detailed analysis of the cost of the scheme for an interval $t$ that belongs to $\operatorname{TreeKU}(i)$ with $2^{i}-i \leq t \leq 2^{i+1}-i-2$ :

1. The center state includes all the root keys of the previous $i-1$ trees and the center state for node $t$ in $\operatorname{TreeKU}(i)$. In the worst-case, this equals $(i-1)+(2 i-1)=$ $3 i-2=3\left\lceil\log _{2}(t)\right\rceil-2$ tree-keys. Similarly, the user key for interval $t$ includes the user key of node $t$ as in scheme $\operatorname{TreeKU}(i)$ and the root keys of the previous $i-1$ trees, in total $(i-1)+(i-1)=2 i-2=2\left\lceil\log _{2}(t)\right\rceil-2$ tree-keys. It follows that the space complexity of the center state and the user key for interval $t$ is $\mathcal{O}\left(\kappa \log _{2}(t)\right)$ bits. 
2. The cost of both Update and Extract algorithms is at most $i=\left\lceil\log _{2}(t)\right\rceil$ applications of the pseudorandom generator. The cost of Derive is constant, as in the tree construction.

\section{Performance of the Constructions}

In this section we analyze the time complexity of the cryptographic operations and the space complexity of the center and the user for the three proposed constructions. Recall that all schemes generate keys of length $\kappa$. In analyzing the time complexity of the algorithms, we specify what kind of operations we measure and distinguish public-key operations (PK op.) from pseudorandom generator applications (PRG op.) because PK operations are typically much more expensive than PRG applications. We omit the time complexity of the Init algorithm, as it involves only the pseudorandom generator for all schemes except for the trapdoor permutation scheme, in which Init also generates the trapdoor permutation. The space complexities are measured in bits. Table 1 shows the details for a given number $T$ of time intervals.

Table 1. Worst-case time and space complexities of the constructions for $T$ time intervals. ${ }^{*}$ Note: the amortized complexity of Update $\left(t, S_{t}\right)$ in the binary tree scheme is constant.

\begin{tabular}{|c|c|c|c|}
\hline & $\mathrm{CKU}$ & TDKU & TreeKU \\
\hline Update $\left(t, S_{t}\right)$ time & 0 & 1 PK op. & $\mathcal{O}\left(\log _{2} T\right)$ PRG op. ${ }^{*}$ \\
\hline Derive $\left(t, S_{t}\right)$ time & $T-t$ PRG op. & const. & $O\left(\log _{2} T\right)$ \\
\hline $\operatorname{Extract}\left(t, M_{t}, i\right)$ time & $t-i$ PRG op. & $t-i$ PK op. & $\mathcal{O}\left(\log _{2} T\right)$ PRG op. \\
\hline Center state size & $\kappa$ & poly $(\kappa)$ & $\overline{\mathcal{O}}\left(\kappa \log _{2} T\right)$ \\
\hline User key size & $\kappa$ & $\kappa$ & $\mathcal{O}\left(\kappa \log _{2} T\right)$ \\
\hline
\end{tabular}

In the chaining scheme CKU, the Update algorithm takes no work, but the Extract and the Derive algorithms take linear work in the number of time intervals. On the other hand, the trapdoor permutation scheme TDKU has efficient user-key derivation, which involves only a copy operation, but the complexity of the Update algorithm is one application of the trapdoor permutation inverse and that of the Extract $\left(t, M_{t}, i\right)$ algorithm is $t-i$ applications of the trapdoor permutation. The tree-based scheme TreeKU balances the tradeoffs between the complexity of the three algorithms, taking logarithmic work in the number of time intervals for all three algorithms in the worst-case. The Derive algorithm involves only $\mathcal{O}\left(\log _{2} T\right)$ copy operations, and Update and Extract algorithms involve $\mathcal{O}\left(\log _{2} T\right)$ PRG operations. This comes at the cost of increasing the center-state and user-key sizes to $\mathcal{O}\left(\kappa \log _{2} T\right)$. Note that the amortized cost of the Update algorithm in the binary tree construction is constant.

As the chaining and the trapdoor permutation schemes have worst-case complexities linear in $T$ for at least one algorithm, both of them require the number of time intervals to be rather small. In contrast, the binary tree construction can be used for a practically unbounded number of time intervals.

In an application in which the number of time intervals in not known in advance, the incremental tree scheme can be used. Its space and time complexities only depend on 
the number of past revocations and not on the total number of revocations supported. The incremental tree construction is an interesting example of an additive composition of tree constructions with different number of intervals. Furthermore, our additive and multiplicative composition methods allow the construction of new schemes starting from the basic three constructions described in Section 4

\section{Experimental Evaluation}

We have implemented the chaining, trapdoor, and tree constructions for 128-bit keys. We have used the 128-bit AES block cipher to implement the pseudorandom generator $G$ as $G(s)=A E S_{s}\left(0^{128}\right)|| A E S_{s}\left(1^{128}\right)$ with $|s|=128$ for the CKU and TreeKU constructions of Sections 4.1 and 4.3. In construction TDKU from Section 4.2, we have used the RSA permutation with a bit length of 1024 and public exponent 3 and the SHA-1 hash function as the random oracle $h$.

We performed the following experiment. For a fixed total number of revocations $T$, the center first initializes the key-updating scheme. Then, the steps below are repeated for $t=1, \ldots, T$ :

- The center runs the Update and Derive algorithms to simulate one revocation.

- Given the user key for interval $t$, the user runs the Extract algorithm to obtain the key $k_{1}$ for the first time interval.

Note that the time to extract the key for the first interval is larger than the extraction time for any other interval between 1 and $t$ in all three constructions. Hence, the extraction time for the first interval represents a worst-case measure. We measured the performance using four metrics: the maximum and average Update and Derive time for the center (over the $T$ revocations), and the maximum and average Extract time for clients to compute the key for the first time interval (from one of the $T$ time intervals). We ran our experiments on a $2.4 \mathrm{GHz}$ Intel Xeon processor machine, running Linux 2.6. Our unoptimized implementation was written in C++ using gcc 3.2.1.

The results are presented in Figures 7, 8, and 9, respectively. The graphs show the measured time as a function of the total number of revocations $T$, which ranges from $2^{8}$ to $2^{25}$ depending on the scheme. Note that the both axis are logarithmic and that the vertical axis differs for the three constructions. In the chaining construction, the cost of

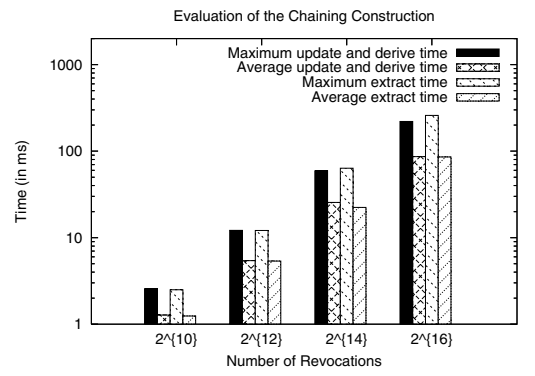

Fig. 7. Evaluation of the chaining scheme

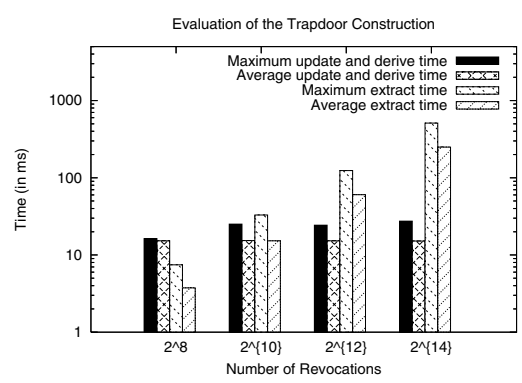

Fig. 8. Evaluation of the trapdoor scheme 


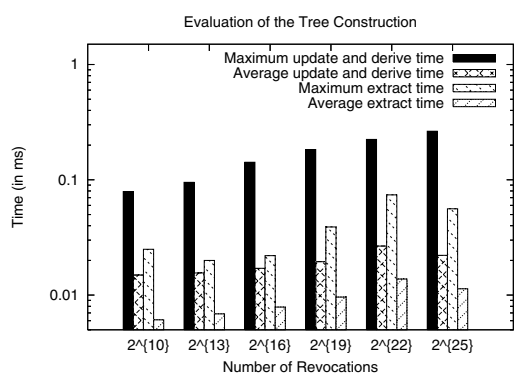

Fig. 9. Evaluation of the tree scheme

both the center and client computation increases linearly with the total number of revocations, as expected. In the trapdoor permutation construction, the center time is always constant, but the extraction time grows linearly with the total number of revocations. In the tree construction, all four metrics have a logarithmic dependence on the total number of revocations. We observe that the tree construction performs several orders of magnitude better than the other schemes.

Table 2 gives a direct comparison of the constructions in an experiment with 1024 revocations as above. It contains also the timing measurements for the first 1024 revocations in the tree construction where the upper bound $T$ on number of revocations was set to a much larger value. This makes it possible to relate the tree construction to the trapdoor permutation scheme, which has no fixed upper bound on the number of revocations. It is evident that the tree scheme performs much better than the other schemes, even with a bound on the number of revocations that allows a practically unlimited number of them.

Table 2. Evaluation of the three constructions for 1024 revocations

\begin{tabular}{|c|c|c|c|c|c|}
\hline Scheme & $T$ & $\begin{array}{c}\text { Maximum Time } \\
\text { Update+Derive }(\mathrm{ms})\end{array}$ & $\begin{array}{c}\text { Average Time } \\
\text { Update+Derive }(\mathrm{ms})\end{array}$ & $\begin{array}{c}\text { Maximum Time } \\
\text { Extract }(\mathrm{ms})\end{array}$ & $\begin{array}{c}\text { Average Time } \\
\text { Extract }(\mathrm{ms})\end{array}$ \\
\hline Chaining & 1024 & 2.57 & 1.28 & 2.5 & 1.24 \\
\hline Trapdoor & 1024 & 25.07 & 15.36 & 32.96 & 15.25 \\
\hline Tree & 1024 & 0.079 & 0.015 & 0.025 & 0.006 \\
\hline Tree & $2^{16}$ & 0.142 & 0.015 & 0.018 & 0.0076 \\
\hline Tree & $2^{25}$ & 0.199 & 0.015 & 0.02 & 0.01 \\
\hline
\end{tabular}

The space usage for $T=1024$ is as follows. The center state is 16 bytes for the chaining construction, 384 bytes for the trapdoor construction, and at most 328 bytes for the tree scheme. The size of the user key is 32 bytes for the chaining construction, 128 bytes for the trapdoor construction, and at most 172 bytes for the tree scheme. In general, for the tree scheme with depth $d$, the center state takes at most $(2 d-1)(16+$ $d / 8$ ) bytes, containing $2 d-1$ key value/key label pairs, assuming 16-byte keys and $d$-bit labels. The user key size is at most $d$ key/label pairs, which take $d(16+d / 8)$ bytes. 
In summary, we note that the performance of the tree scheme is superior to the others. The chaining construction has the smallest space requirements, but its computation cost becomes prohibitive for large $T$. The trapdoor construction has sligthly smaller space requirements than the tree scheme, but these savings are very small compared to the additional computational overhead.

\section{Related Work}

Time-Evolving Cryptography. The notion of secure key-updating schemes is closely related to forward- and backward-secure cryptographic primitives. Indeed, a secure keyupdating scheme is forward-secure as defined originally by Anderson [4], in the sense that it maintains security in the time intervals following a key exposure. However, this is the opposite of the forward security notion formalized by Bellare and Miner [7] and used in subsequent work. Here we use the term forward security to refer to the latter notion.

Time-evolving cryptography protects a cryptographic primitive against key exposure by dividing the time into intervals and using a different secret key for every time interval. Forward-secure primitives protect past uses of the secret key: if a device holding all keys is compromised, the attacker can not have access to past keys. In the case of forward-secure signatures, the attacker can not generate past signatures on behalf of the user, and in the case of forward-secure encryption, the attacker can not decrypt old ciphertexts. There exist many efficient constructions of forward-secure signatures [7|2|21] and several generic constructions [24]26]. Bellare and Yee [8] analyze forward-secure private-key cryptographic primitives (forward-secure pseudorandom generators, message authentication codes and symmetric encryption) and Canetti, Halevi and Katz [10] construct the first forward-secure public-key encryption scheme.

Forward security has been combined with backward security in models that protect both the past and future time intervals, called key-insulated [13 14] and intrusionresilient models [22|12]. In both models, there is a center that interacts with the user in the key update protocol. The basic key insulation model assumes that the center is trusted and the user is compromised in at most $t$ time intervals and guarantees that the adversary does not gain information about the keys for the intervals the user is not compromised. A variant of this model, called strong key insulation, allows the compromise of the center as well. Intrusion-resilience tolerates arbitrarily many break-ins into both the center and the user, as long as the break-ins do not occur in the same time interval. The relation between forward-secure, key-insulated and intrusion-resilient signatures has been analyzed by Malkin, Obana and Yung [27]. A survey of forward-secure cryptography is given by Itkis [20].

Re-keying, i.e., deriving new secret keys periodically from a master secret key, is a standard method used by many applications. It has been formalized by Abdalla and Bellare [1]. The notion of key-updating schemes that we define is closely related to re-keying schemes, with the difference that in our model, we have the additional requirement of being able to derive past keys efficiently.

Multicast Key Distribution. In key distribution schemes for multicast, a group controller distributes a group encryption key to all users in a multicast group. The group of users 
is dynamic and each join or leave event requires the change of the encryption key. The goal is to achieve both forward and backward security. In contrast, in our model of key-updating schemes users should be able to derive past encryption keys efficiently.

A common key distribution model for multicast is that of key graphs, introduced by Wong et al. [32] and used subsequently in many constructions [30], [29], [19],[18]. In these schemes, each user knows its own secret key and, in addition, a subset of secret keys used to generate the group encryption key and to perform fast update operations. The relation between users and keys is modeled in a directed acyclic graphs, in which the source nodes are the users, intermediary nodes are keys and the unique sink node is the group encryption key. A path from a user node to the group key contains all the keys known to that user. The complexity and communication cost of key update operations is optimal for tree structures [31], and in this case it is logarithmic in the number of users in the multicast group. We also use trees for generating keys, but our approach is different in considering the nodes of the tree to be only keys, and not users. We obtain logarithmic update cost in the number of revocations, not in the number of users in the group.

Key Management in Cryptographic Storage Systems. Early cryptographic file systems [911] did not address key management. Cepheus [15] is the first cryptographic file system that considers sharing of files and introduces the idea of lazy revocation for improving performance. However, key management in Cepheus is centralized by using a trusted key server for key distribution. More recent cryptographic file systems, such as Oceanstore [25] and Plutus [23], acknowledge the benefit of decentralized key distribution and propose that key management is handled by file owners themselves. For efficient operation, Plutus adopts a lazy revocation model and uses a key-updating scheme based on RSA, as described in Section 4.2 .

Farsite [3], SNAD [28] and SiRiUS [17] use public-key cryptography for key management. The group encryption key is encrypted with the public keys of all group members and these lockboxes are stored on the storage servers. This approach simplifies key management, but the key storage per group is proportional to the number of users in the group. Neither of these systems addresses efficient user revocation.

Independently and concurrently to our work Fu, Kamara, and Kohno [16] have proposed a cryptographic definition for key-updating schemes, which they call key regression schemes. Key regression schemes are, in principle, equivalent to key-updating schemes. Their work formalizes three key regression schemes: two constructions, one using a hash function and one using a pseudo-random permutation, are essentially equivalent to our chaining construction, and an RSA-based construction originating in Plutus, which is equivalent to our trapdoor-permutation construction. Our composition methods and the tree-based construction are novel contributions that go beyond their work.

\section{Conclusions}

Motivated by the practical problem of efficient key management for cryptographic file systems that adopt lazy revocation, we define formally the notion of key-updating schemes for lazy revocation and its security. In addition, we give two methods for additive and multiplicative composition of two secure key-updating scheme into a new 
scheme which can handle a larger number of user revocations, while preserving security. We also prove the security of two slightly modified existing constructions and propose a new construction, the binary-tree scheme, that balances the tradeoffs of the existing constructions. Finally, we provide a systematic analysis of the computational and communication complexities of the three constructions.

We can extend the definition of key-updating schemes to support user keys for interval $t$, from which only keys of the time intervals between $i$ and $t$ can be extracted, for any $1 \leq i \leq t$. This is useful in a model in which users joining the group at a later time interval should not have access to past information. The extension can be incorporated in the tree construction without additional cost, but the chaining and trapdoor permutation constructions do not work in this model because the user key reveals all previous keys.

In a companion paper [5], we show how to extend secure key-updating schemes to cryptosystems with lazy revocation, and introduce the notions of symmetric encryption, message-authentication codes, and signature schemes with lazy revocation. Furthermore, we demonstrate that using these cryptosystems in some existing distributed cryptographic file systems improves their efficiency and security.

\section{References}

1. M. Abdalla and M. Bellare, "Increasing the lifetime of a key: A comparitive analysis of the security of rekeying techniques," in Proc. Asiacrypt 2000, vol. 1976 of Lecture Notes in Computer Science, pp. 546-559, Springer-Verlag, 2000.

2. M. Abdalla and L. Reyzin, "A new forward-secure digital signature scheme," in Proc. Asiacrypt 2000, vol. 1976 of Lecture Notes in Computer Science, pp. 116-129, Springer-Verlag, 2000.

3. A. Adya, W. J. Bolosky, M. Castro, G. Cermak, R. Chaiken, J. R. Douceur, J. Howell, J. R. Lorch, M. Theimer, and R. P. Wattenhofer, "FARSITE: Federated, available, and reliable storage for an incompletely trusted environment," in Proc. 5th Symposium on Operating System Design and Implementation (OSDI), Usenix, 2002.

4. R. Anderson, "Two remarks on public-key cryptology," Technical Report UCAM-CL-TR549, University of Cambridge, 2002.

5. M. Backes, C. Cachin, and A. Oprea, "Lazy revocation in cryptographic file systems," in Proc. 3rd Intl. IEEE Security in Storage Workhsop (SISW), 2005.

6. M. Backes, C. Cachin, and A. Oprea, "Secure key-updating for lazy revocation," Research Report RZ 3627, IBM Research, Aug. 2005. Appears also as Cryptology ePrint Archive, Report 2005/334.

7. M. Bellare and S. Miner, "A forward-secure digital signature scheme," in Proc. Crypto 1999, vol. 1666 of Lecture Notes in Computer Science, pp. 431-448, Springer-Verlag, 1999.

8. M. Bellare and B. Yee, "Forward-security in private-key cryptography," in Proc. CT-RSA 2003, vol. 2612 of Lecture Notes in Computer Science, pp. 1-18, Springer-Verlag, 2003.

9. M. Blaze, "A cryptographic file system for Unix," in Proc. First ACM Conference on Computer and Communication Security (CCS), pp. 9-16, 1993.

10. R. Canetti, S. Halevi, and J. Katz, "A forward-secure public-key encryption scheme," in Proc. Eurocrypt 2003, vol. 2656 of Lecture Notes in Computer Science, pp. 255-271, SpringerVerlag, 2003.

11. G. Cattaneo, L. Catuogno, A. D. Sorbo, and P. Persiano, "The design and implementation of a transparent cryptographic file system for Unix," in Proc. USENIX Annual Technical Conference 2001, Freenix Track, pp. 199-212, 2001. 
12. Y. Dodis, M. Franklin, J. Katz, A. Miyaji, and M. Yung, "Intrusion-resilient public-key encryption," in Proc. CT-RSA 2003, vol. 2612 of Lecture Notes in Computer Science, pp. 19-32, Springer-Verlag, 2003.

13. Y. Dodis, J. Katz, S. Xu, and M. Yung, "Key insulated public-key cryptosystems," in Proc. Eurocrypt 2002, vol. 2332 of Lecture Notes in Computer Science, pp. 65-82, SpringerVerlag, 2002.

14. Y. Dodis, J. Katz, and M. Yung, "Strong key-insulated signature schemes," in Proc. Workshop of Public Key Cryptography (PKC), vol. 2567 of Lecture Notes in Computer Science, pp. 130-144, Springer-Verlag, 2002.

15. K. Fu, "Group sharing and random access in cryptographic storage file systems," Master's thesis, Massachusetts Institute of Technology, 1999.

16. K. Fu, S. Kamaram, and T. Kohno, "Key regression: Enabling efficient key distribution for secure distributed storage," in Proc. Network and Distributed Systems Security Symposium (NDSS 2006), 2006.

17. E. Goh, H. Shacham, N. Modadugu, and D. Boneh, "SiRiUS: Securing remote untrusted storage," in Proc. Network and Distributed Systems Security Symposium (NDSS 2003), pp. 131$145,2003$.

18. M. T. Goodrich, J. Z. Sun, and R. Tamassia, "Efficient tree-based revocation in groups of low-state devices," in Proc. Crypto 2004, vol. 3152 of Lecture Notes in Computer Science, pp. 511-522, Springer-Verlag, 2004.

19. J. Goshi and R. E. Ladner, "Algorithms for dynamic multicast key distribution trees," in Proc. 22nd Symposium on Principles of Distributed Computing (PODC), pp. 243-251, ACM, 2003.

20. G. Itkis, "Forward security, adaptive cryptography: Time evolution." Survey, available from http: / / www.cs.bu.edu/fac/itkis/pap/forward-secure-survey.pdf.

21. G. Itkis and L. Reyzin, "Forward-secure signatures with optimal signing and verifying," in Proc. Crypto 2001, vol. 2139 of Lecture Notes in Computer Science, pp. 332-354, SpringerVerlag, 2001.

22. G. Itkis and L. Reyzin, "SiBIR: Signer-base intrusion-resilient signatures," in Proc. Crypto 2002, vol. 2442 of Lecture Notes in Computer Science, pp. 499-514, Springer-Verlag, 2002.

23. M. Kallahalla, E. Riedel, R. Swaminathan, Q. Wang, and K. Fu, "Plutus: Scalable secure file sharing on untrusted storage," in Proc. 2nd USENIX Conference on File and Storage Technologies (FAST), 2003.

24. H. Krawczyk, "Simple forward-secure signatures from any signature scheme," in Proc. 7th ACM Conference on Computer and Communication Security (CCS), pp. 108-115, 2000.

25. J. Kubiatowicz, D. Bindel, Y. Chen, S. Czerwinski, P. Eaton, D. Geels, R. Gummadi, S. Rhea, H. Weatherspoon, W. Weimer, C. Wells, and B. Zhao, "Oceanstore: An architecture for global-scale persistent storage," in Proc. 9th International Conference on Architectural Support for Programming Languages and Operating Systems (ASPLOS), pp. 190-201, ACM, 2000.

26. T. Malkin, D. Micciancio, and S. Miner, "Efficient generic forward-secure signatures with an unbounded number of time periods," in Proc. Eurocrypt 2002, vol. 2332 of Lecture Notes in Computer Science, pp. 400-417, Springer-Verlag, 2002.

27. T. Malkin, S. Obana, and M. Yung, "The hierarchy of key evolving signatures and a characterization of proxy signatures," in Proc. Eurocrypt 2004, vol. 3027 of Lecture Notes in Computer Science, pp. 306-322, Springer-Verlag, 2004.

28. E. Miller, D. Long, W. Freeman, and B. Reed, "Strong security for distributed file systems," in Proc. the First USENIX Conference on File and Storage Technologies (FAST), 2002.

29. O. Rodeh, K. Birman, and D. Dolev, "Using AVL trees for fault tolerant group key management," International Journal on Information Security, vol. 1, no. 2, pp. 84-99, 2001. 
30. A. T. Sherman and D. A. McGrew, "Key establishment in large dynamic groups using oneway function trees," IEEE Transactions on Software Engineering, vol. 29, no. 5, pp. 444-458, 2003.

31. R. Tamassia and N. Triandopoulos, "Computational bounds on hierarchical data processing with applications to information security," in Proc. 32nd International Colloquium on Automata, Languages and Programming (ICALP), 2005.

32. C. K. Wong, M. Gouda, and S. S. Lam, "Secure group communications using key graphs," IEEE/ACM Transactions on Networking, vol. 8, no. 1, pp. 16-30, 2000. 